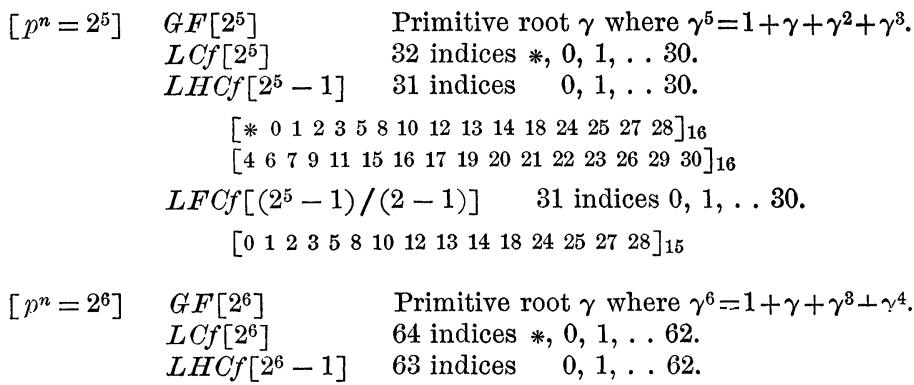

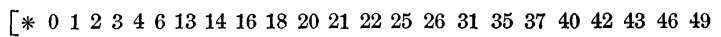
$\left.\begin{array}{llllllll}50 & 51 & 53 & 54 & 56 & 57 & 58 & 59\end{array}\right]_{32}$

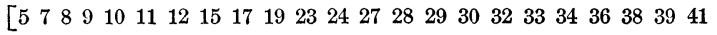

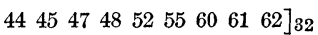

$\operatorname{LFCf}\left[\left(2^{6}-1\right) /(2-1)\right] \quad 63$ indices $0,1, \ldots 62$.

[First line above, omitting the $*]_{31}$

The University of Chichao, August 25, 1895.

\title{
ELEMENTARY PROOF OF THE QUATERNION ASSOCIATIVE PRINCIPLE.*
}

\section{BY PROFESSOR ARTHUR S. HATHAWAY.}

The variety of demonstrations that Hamilton has given of the associative principle of quaternion multiplication, and the remarks that he has made upon such demonstrations, show that he considered an elementary proof of this principle as very desirable. Only two of Hamilton's proofs have been generally employed by subsequent writers - the direct proof by spherical conics, and the indirect one depending upon the assumed laws of $i, j, k$-and the proof that he considered the most elementary has been entirely ignored, probably because of its deviation from fundamental ideas. On page 297 of the Elements, Hamilton calls attention to another method, as follows : "The associative principle of multiplication may also be proved without the distributive principle, by certain considerations of rotations of a system, on which we cannot enter here."

It is, of course, easy to see that such a proof is possible; but the details of it could not have presented themselves to Hamilton in an elementary form, or he would have seen that it

* Presented to the American Mathematical Society August 28, 1895. 
was just the demonstration for which he was looking, simple in character, and direct in its application.

The foundation consists of a few simple data with regard to the rotation of a sphere about its diameters, and a lemma that immediately identifies versor multiplication with the composition of rotations.

By the rotation, 2 arc $q$, we mean the rotation whose equator, angular magnitude, and direction, are given by double the are that gives the plane, angular magnitude, and direction, of the turning or versor operation of $q$. The axis of $q$ is therefore the axis of the rotation; and as arc $q$ is not greater than a semicircumference, so 2 arc $q$ is not greater than a circumference. There is not more than one axis around which the sphere may be rotated from one position to another, since any diameter that is oblique to one axis returns to its original position only for entire revolutions of the sphere, and cannot therefore be another axis of rotation. Hence there is not more than one equator pertaining to the two positions of the sphere, and all arcs that specify rotations between those positions must lie upon the same great circle and be equal in magnitude and direction to one or the other of two oppositely directed arcs whose combined lengths make a circumference. The halves of any two such arcs are therefore either equal and concurrent, or supplementary and opposite.

Lemma. The rotation 2 arc $r$, followed by the rotation 2 arc $q$, produces the same displacement of the sphere as the single rotation 2 arc $q r$.

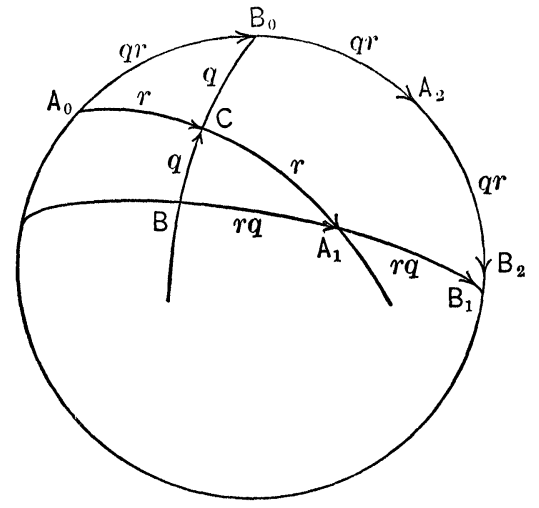

For let $C$ be a point of intersection of the great circles of $r$ and $q$, and lay off the given arcs, arc $r=A_{0} C=C A_{1}$, arc $q$ $=B^{\prime} C=C B_{0}$; and also construct arc $r q=B^{\prime} A_{1}=A_{1} B_{1}$, and arc $q r=A_{0} B_{0}=B_{0} A_{2}=A_{2} B_{2}$. By construction, the spherical tri- 
angles $A_{0} B_{0} C, A_{1} B^{\prime} C$, are equal; and therefore the arcs $A_{0} B_{0}$, $A_{1} B_{1}$, are equal and equally inclined to the arc $A_{0} A_{1}$, and the continuous arcs $B^{\prime} A_{1}, A_{1} B_{1}$, are equal to the continuous arcs $B_{0} A_{2}, A_{2} B_{2}$. and these two continuous arcs are equally inclined to $B^{\prime} B_{0}$. Hence, if a marked arc $A B$ of the sphere initially coincide with $A_{0} B_{0}$, then the rotation of the sphere through 2 arc $r=A_{0} A_{1}$, brings $A B$ into the position $A_{1} B_{1}$, and the next rotation through 2 arc $q=B^{\prime} B_{0}$, brings $A B$ into the position $A_{2} B_{2}$. From the figure it is obvious that the position $A_{2} B_{2}$ is reached from the position $A_{0} B_{0}$ by the single rotation 2 arc $q r$ $=A_{0} A_{2}$.

This lemma shows that the successive rotations, 2 arc $r$, 2 arc $q, 2$ arc $p$, produce the same displacement of the sphere as either of the single rotations, 2 arc $p(q r)$, or $2 \operatorname{arc}(p q) r$ (according as we begin by compounding the first and second, or the second and third, rotations into their equivalent single rotations). Hence, $\operatorname{arc} p(q r)$ and $\operatorname{arc}(p q) r$ are equal and concurrent, or supplementary and opposite arcs of the same great circle. We may rely upon a diagram to show the currency of these two arcs, and therefore their complete versor equality, or we may prove the same by the principles of continuity, as follows: When $p, q, r$, change continuously in value, their arcs and the arcs of their products also change continuously in circle length and direction. Hence, if $\operatorname{arc} p(q r)$ and $\operatorname{arc}(p q) r$ are equal and concurrent for particular values of $p, q, r$ (as is obviously the case), they must continue so for all values of $p, q, r$, since an indefinitely small change in each arc cannot change them from equal concurrent ares to the only other possible case of supplementary opposite arcs.

So in general, if the sphere receive the successive rotations, $2 \operatorname{arc} r, 2 \operatorname{arc} q, 2 \operatorname{arc} p, \ldots$, then its displacement is the same as by the single rotation, 2 arc (...pqr), in whatever way the factors of this product are associated to form such product. Therefore the arcs of any two such products are either equal and concurrent, or supplementary and opposite arcs of the same great circle; and the principles of continuity, and the special case in which each arc begins where the preceding arc ends, show that the first-named relation is the true one. Since the tensors of all such products are also equal, therefore all such products, formed by different associations of the same factors in the same order, are equal.

Rose Polytechnic Institute, Terre Haute, InD. 